\title{
Combined treatment of Pseudomonas aeruginosa biofilm with lactoferrin and xylitol inhibits the ability of bacteria to respond to damage resulting from lactoferrin iron chelation
}

\author{
Mary Cloud B. Ammons ${ }^{\mathrm{a}, *}$, Loren S. Ward ${ }^{\mathrm{b}}$, Scot Dowd ${ }^{\mathrm{c}}$, Garth A. James ${ }^{\mathrm{a}}$ \\ a Center for Biofilm Engineering, 366 EPS Building, Montana State University, Bozeman, MT 59717, USA \\ ${ }^{\mathrm{b}}$ Glanbia Research and Development Center, 450 Falls Avenue, Suite 255, Twin Falls, ID 83301, USA \\ ${ }^{\mathrm{c}}$ Medical Biofilm Research Institute, Southwest Regional Wound Care Center, 2002 Oxford Ave., Lubbock, TX 79410, USA
}

\section{A R T I C L E I N F O}

\section{Article history:}

Received 9 June 2010

Accepted 22 December 2010

\section{Keywords:}

Biofilm

Lactoferrin

Xylitol

Pseudomonas aeruginosa

\begin{abstract}
A B S T R A C T
With an ageing and ever more obese population, chronic wounds such as diabetic ulcers, pressure ulcers and venous leg ulcers are an increasingly relevant medical concern. Identification of bacterial biofilm contamination as a major contributor to non-healing wounds demands biofilm-targeted strategies to manage chronic wounds. Pseudomonas aeruginosa has been identified as a principal biofilm-forming opportunistic pathogen in chronic wounds. The innate immune molecule lactoferrin and the rare sugar alcohol xylitol have been demonstrated to be co-operatively efficacious against $P$. aeruginosa biofilms in vitro. Data presented here propose a model for the molecular mechanism behind this co-operative antimicrobial effect. Lactoferrin iron chelation was identified as the primary means by which lactoferrin destabilises the bacterial membrane. By microarray analysis, 183 differentially expressed genes of $\geq 1.5$-fold difference were detected. Interestingly, differentially expressed transcripts included the operon encoding components of the pyochelin biosynthesis pathway. Furthermore, siderophore detection verified that xylitol is the component of this novel synergistic treatment that inhibits the ability of the bacteria to produce siderophores under conditions of iron restriction. The findings presented here demonstrate that whilst lactoferrin treatment of $P$. aeruginosa biofilms results in destabilisation of the bacterial cell membrane though iron chelation, combined treatment with lactoferrin and xylitol inhibits the ability of $P$. aeruginosa biofilms to respond to environmental iron restriction.
\end{abstract}

(C) 2011 Elsevier B.V. and the International Society of Chemotherapy. All rights reserved.

\section{Introduction}

With the epidemic rise in diabetes and an ageing population, chronic wounds are an increasingly relevant medical concern. Chronic wounds include diabetic foot ulcers, pressure ulcers and venous leg ulcers and result in ca. $100000 \mathrm{limb}$ amputations each year in the USA [1]. Chronicity in wounds appears to be initiated by persistent levels of contaminating bacteria resulting in chronic inflammation [2,3]. Much of the contaminating bacteria in a nonhealing wound are organised into structured communities known as biofilms [1], leading to a lack of wound resolution by the host and development of bacterial resistance to antibiotic therapy [4]. With the verification that bacterial biofilm contamination is a major contributor to non-healing wounds, there is clearly a need for strategies that target biofilms in the management of chronic wounds [5].

\footnotetext{
* Corresponding author. Tel.: +1 406994 7962; fax: +1 4069946098

E-mail address: mary.ammons@erc.montana.edu (M.C.B. Ammons).
}

Chronic wounds primarily afflict mildly immunocompromised individuals and thus the contaminating bacteria typically involve opportunistic pathogens, including the aquatic bacterium Pseudomonas aeruginosa [4]. Although not a common pathogen of individuals with a healthy immune system, $P$. aeruginosa has been demonstrated to be an opportunistic pathogen in ear infections [6], chronic bacterial prostatitis [7,8] and, most commonly, cystic fibrosis [9]. It is estimated that over one-half of all chronic wounds are colonised by $P$. aeruginosa [10]. Indeed, a molecular analysis of chronic wounds found $P$. aeruginosa to be a dominant species in venous leg ulcers as well as pseudomonads to be a top species in diabetic foot ulcers [11]. These data substantiate that $P$. aeruginosa is a commonly found pathogen of chronic wounds and should be targeted for wound therapy.

Lactoferrin is an iron-chelating protein of the innate immune system found in most bodily fluids such as blood, saliva and tears (reviewed in [12]). In addition to modulating the immune system, lactoferrin has been demonstrated to act as an antimicrobial. For example, lactoferrin has been demonstrated to inhibit $P$. aeruginosa biofilm formation by triggering bacterial motility [13]. Another 
naturally occurring antimicrobial is the rare sugar alcohol xylitol, which can be found in small quantities in fruit and vegetables [14].Xylitol treatment was recently demonstrated to be moderately effective at inhibiting biofilm growth of the opportunistic pathogen P. aeruginosa [15].

Although both lactoferrin and xylitol have been demonstrated independently as antibiofilm therapies, both have also been shown to enhance antimicrobial effects when used in combination with other therapies. An antibiofilm co-operative effect on $P$. aeruginosa biofilms with combined lactoferrin and xylitol treatment was recently demonstrated [15]. Although lactoferrin and xylitol combined treatment was demonstrated to be more efficacious than use of either antimicrobial independently, the mechanism of action responsible was not investigated.

This study reports on the molecular mechanism contributing to the synergistic effect of using a combination of lactoferrin and xylitol to inhibit P. aeruginosa biofilms and proposes a model in which xylitol inhibition of the pyochelin biosynthesis pathway enhances the antimicrobial activity of lactoferrin.

\section{Methods}

\subsection{Bacteria and media}

All assays used the clinical wound isolate $P$. aeruginosa 215 obtained by wound biopsy from the Southwest Regional Wound Clinic (Lubbock, TX). Pseudomonas aeruginosa 215 frozen stock was cultured overnight in $10 \%$ brain-heart infusion (BHI) broth at $37^{\circ} \mathrm{C}$ with shaking. Overnight $P$. aeruginosa 215 culture was used to inoculate Centers for Disease Control and Prevention (CDC) Biofilm Reactors (BioSurface Technologies Inc., Bozeman, MT) containing $10 \% \mathrm{BHI}$ broth at room temperature. For the siderophore detection assay, overnight $P$. aeruginosa 215 culture was used to inoculate King's B (KB) medium without phosphate (magnesium sulphate $1.5 \mathrm{~g} / \mathrm{L}$, tryptone $10 \mathrm{~g} / \mathrm{L}$, protease peptone $10 \mathrm{~g} / \mathrm{L}$ and glycerol $10 \mathrm{~mL} / \mathrm{L}$ ).

\subsection{Biofilm growth conditions}

Pseudomonas aeruginosa 215 was grown in CDC reactors as described in ASTM standard \#E2562-07 [16], with some modifications. Sterilised CDC reactors containing $500 \mathrm{~mL}$ of $10 \% \mathrm{BHI}$ were inoculated with $P$. aeruginosa 215 overnight culture. A 24-h batch, with stirring, allowed establishment of biofilms on coupons prior to initiation of flow at $2.7 \mathrm{~mL} / \mathrm{min}$. CDC reactors were then run in flow mode for $24 \mathrm{~h}$. Coupons were then harvested from the CDC reactors and were collected into $10 \mathrm{~mL}$ of sterile phosphate-buffered saline (PBS). To disaggregate bacterial cells, the coupons were vortexed for $10 \mathrm{~s}$, sonicated for $2 \mathrm{~min}$ and then vortexed again for $10 \mathrm{~s}$. For viability assays, the disaggregated bacterial cells were serially diluted and plated on $100 \%$ tryptic soy agar (TSA). Colony-forming units were then counted and the log density of viable bacterial cells was calculated. Data were reported as log reduction relative to the log density calculated for the control sample. For planktonic samples, bacterial cells were recovered from CDC chamber effluent, diluted into sterile PBS, vortexed and sonicated as above, and then serially diluted and plated on $100 \%$ TSA. Viability was calculated as above.

For membrane permeabilisation assays, CDC reactors were treated with $2 \%(\mathrm{w} / \mathrm{v})$ lactoferrin (Bioferrin ${ }^{\circledR}$; Glanbia Nutritionals Inc., Monroe, WI) and/or 5\% (w/v) xylitol (Sigma-Aldrich, St Louis, $\mathrm{MO}$ ) added to the flow medium (10\% BHI). For the endotoxin assays, lactoferrin containing either low levels of endotoxin $(<1 \mu \mathrm{g} / \mathrm{mg}$ lactoferrin) or high levels of endotoxin ( $>500 \mu \mathrm{g} / \mathrm{mg}$ lactoferrin) were added to the flow medium $(10 \% \mathrm{BHI})$ at a concentration of $2 \%$ $(\mathrm{w} / \mathrm{v})$. For iron saturation assays, CDC reactors were treated with
$2 \%(\mathrm{w} / \mathrm{v}$ ) lactoferrin pre-saturated with $0.25 \mu \mathrm{M} \mathrm{Fe} / \mathrm{mg}$ lactoferrin added to the flow medium (10\% BHI). For the siderophore detection assays, $500 \mu \mathrm{L}$ of overnight $P$. aeruginosa 215 culture was used to inoculate $500 \mathrm{~mL}$ of $\mathrm{KB}$ medium either with or without added $2 \%$ $(\mathrm{w} / \mathrm{v})$ lactoferrin or $5 \%(\mathrm{w} / \mathrm{v})$ xylitol.

\subsection{Fluorescent staining and imaging}

Cell membrane integrity was assayed using the LIVE/DEAD ${ }^{\circledR}$ BacLight $^{\mathrm{TM}}$ Bacterial Viability Kit (Molecular Probes, Eugene, OR), which enables differentiation between cells with intact versus permeabilised membranes. To visualise and quantitate membrane permeabilisation, $1 \mathrm{~mL}$ of disaggregated biofilm was stained with $6 \mu \mathrm{L}$ of each component prior to adherence to black polycarbonate membranes via vacuum filtration.

Membrane-adhered and stained samples were imaged using a Nikon Eclipse E800 microscope with a 100× oil objective. Images were collected using MetVue software and were analysed using MetaMorph ${ }^{\circledR}$ software (Molecular Devices Corp., Downingtown, PA). For quantification of permeabilised cells, at least three random images were analysed for each stain for at least three coupons per experiment. Data presented are representative of repeat experiments.

\subsection{Microarray analysis}

\subsubsection{Isolation of RNA}

Coupons harvested from CDC reactors were incubated in $1 \mathrm{~mL}$ of Bacterial RNA Protect (QIAGEN, Valencia, CA) for $5 \mathrm{~min}$ at room temperature. To each coupon, $2 \mathrm{~mL}$ of sterile PBS was added and the bacteria were disaggregated as described above. Disaggregated bacteria were pelleted at $6000 \times \mathrm{g}$ for $10 \mathrm{~min}$ at $4{ }^{\circ} \mathrm{C}$. Bacterial pellets were then re-suspended in Tris-ethylene diamine tetra-acetic acid (TE) buffer containing $20 \mathrm{mg} / \mathrm{mL}$ lysozyme (Sigma-Aldrich) and $20 \mathrm{mg} / \mathrm{mL}$ Proteinase K (QIAGEN). Cells were lysed for $30 \mathrm{~min}$ at room temperature with periodic vortexing. RNA was then extracted using the RNeasy Bacterial Mini Kit (QIAGEN) according to the manufacturer's protocol. Gel electrophoresis revealed intact RNA bands and no contaminating genomic DNA. RNA concentration and contamination were determined by measuring absorption at $260 \mathrm{~nm}$ and $280 \mathrm{~nm}$ with a NanoDrop ND-1000 spectrophotometer (Thermo Scientific, Wilmington, DE).

\subsubsection{Microarray hybridisation, scanning and image analysis}

Total pooled RNA from multiple experiments was sent for microarray hybridisation, scanning and image acquisition to the Research and Testing Laboratories at the Medical Biofilm Research Institute (Lubbock, TX). Total RNA was reverse transcribed into cDNA and was labelled with either cyanine 3 or cyanine 5 nucleotide (Perkin Elmer, Wellesley, MA). To account for dye bias, dye swaps were performed for both treatments and control. Microarray hybridisation was performed with whole genome $P$. aeruginosa PAO1 and slides were scanned using a GenePix Personal 4100A Microarray Scanner (Molecular Devices Corp., Sunnyvale, CA). Image acquisition and quantification were performed with GenePix Pro Software v6.0 (Molecular Devices Corp.). Gene expression ratios of $\geq 1.5 \log$ relative to control samples were considered to be differentially expressed. Function and functional category of differentially expressed genes were assigned from the Pseudomonas Genome Database V2 (http://www.pseudomonas.com/search.jsp). Additionally, Venn diagrams of differentially expressed genes were generated using VENNY openware (http://bioinfogp. cnb.csic.es/tools/venny/index.html). Differentially expressed transcripts were compared against microarray analyses deposited at the National Center for Biotechnology Information (NCBI) Gene Expression Omnibus (GEO) (http://ncbi.nlm.nih.gov/geo). 

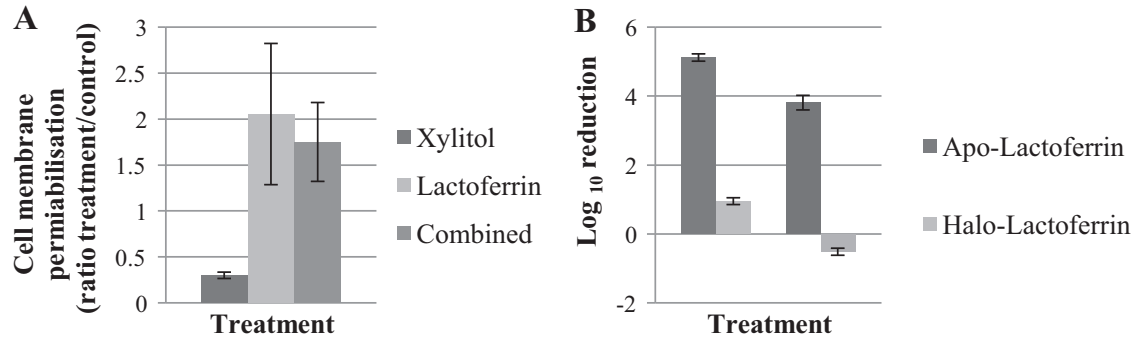

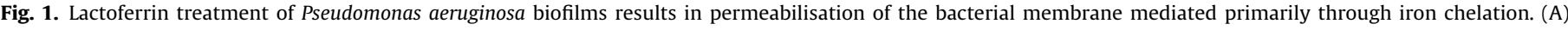

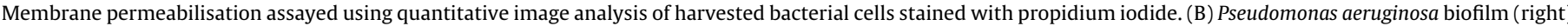

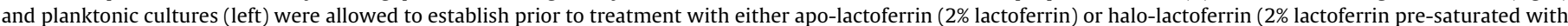

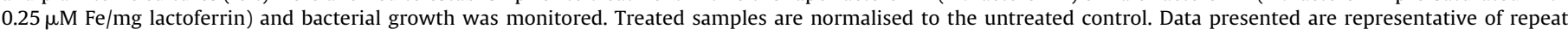
experiments.

\subsection{Real-time quantitative polymerase chain reaction ( $q P C R$ )}

Total RNA was extracted from treated and untreated $P$. aeruginosa grown in CDC reactors as described above. cDNA was generated using the QuantiTect Reverse Transcription Kit (QIAGEN) according to the manufacturer's protocol, including elimination of contaminating genomic DNA. Primers were designed using the Primer-BLAST openware software at the NCBI (http://www.ncbi.nlm.nih.gov/tools/primer-blast/index.cgi).

Primer sequences are given in Table 1 . The specificity of primers was analysed by dissociation curve in the qPCR. qPCR was performed in a Rotor-Gene 6000 Cycler (QIAGEN). QuantiTect SYBR Green PCR Master Mix (QIAGEN) was used to perform real-time PCR. The results obtained were normalised using threshold cycles $\left(C_{T}\right)$ for the endogenous control (16S rRNA) cDNA amplification run on the same plate by using the $\Delta \Delta C_{T}$ method [17]. Cycling conditions included an activation step of $15 \mathrm{~min}$ at $95^{\circ} \mathrm{C}$, followed by 35 cycles of $15 \mathrm{~s}$ at $94^{\circ} \mathrm{C}, 30 \mathrm{~s}$ at $50^{\circ} \mathrm{C}$ and $30 \mathrm{~s}$ at $72^{\circ} \mathrm{C}$, and subsequent data acquisition and melt curve analysis (from $55^{\circ} \mathrm{C}$ to $95^{\circ} \mathrm{C}$ ). Negative controls included non-template controls and no reverse transcriptase controls to exclude contamination or carry-over. Data presented are representative of biological replicates performed in experimental triplicates.

\subsection{Siderophore detection}

Siderophore detection from culture supernatants was performed as described previously $[18,19]$ with some modifications. Briefly, $P$. aeruginosa 215 was cultured as described above in KB medium modified with either $2 \%(\mathrm{w} / \mathrm{v})$ lactoferrin or $5 \%(\mathrm{w} / \mathrm{v})$ xylitol as appropriate or left untreated as control. Growth rate was measured over $48 \mathrm{~h}$ by monitoring turbidity at $600 \mathrm{~nm}$. In pilot experiments, siderophore production was detected at 420 ,

Table 1

Sequences of real-time polymerase chain reaction (PCR) primers.

\begin{tabular}{lll}
\hline Target & Primer & Sequence \\
\hline $16 S$ rRNA & Forward & CAAAACTACTGAGCTAGAGTACG \\
& Reverse & GCCACTGGTGTTCCTCCTA \\
pchR & Forward & TGACCATCACCATCATTGCT \\
& Reverse & CACCAGCTTCATGTTCGGA \\
pchA & Forward & GATCGAGGAAAACTGGCAAT \\
& Reverse & CAGAGTACCCGGTAGCGTTC \\
fptA & Forward & AATCCGTCTACATCAGCGG \\
& Reverse & TCCTTGAGGTACTCGGTGCT \\
napD & Forward & GCCCATGAATGCCTGGCGGT \\
& Reverse & GACATCGGCGGCAAGCAGGT \\
cyoC & Forward & CCCGCAGGGCAAGCTGATCC \\
& Reverse & AGCACGGCGTTGAGCACTCC \\
& Forward & CCACGAAGTGGGCCACGACC \\
& Reverse & AGCACGGCGTTGAGCACTCC \\
\hline
\end{tabular}

630 and $690 \mathrm{~nm}$. Detection at $420 \mathrm{~nm}$ was found to be most consistent over the course of $48 \mathrm{~h}$ in the absence of iron. Samples were taken at the time of inoculation and after $24 \mathrm{~h}$ and $48 \mathrm{~h}$ of growth. Culture supernatants were sterile filtered, diluted 1:1 with $2 \mathrm{mM}$ chrome azurol $\mathrm{S}$ (CAS) reagent (Sigma-Aldrich) and $1 \mathrm{mM} \mathrm{FeCl}_{3}$, and incubated in the dark for $2 \mathrm{~h}$ at room temperature. The CAS assay enables detection of siderophore production and secretion into the medium through a colorimetric assay.

The CAS reagent is a dye that weakly binds iron (III). In the presence of an iron-binding ligand, the dye is stripped of its iron. Release of the free dye is accompanied by a colour change that can be detected at multiple wavelengths. Since lactoferrin is also an iron chelator, detection of siderophores was normalised not only to the growth curves but also to a non-inoculated control medium modified with each respective treatment. Under unmodified conditions, Pseudomonas spp. grown in KB medium produce siderophores peaking at 2 days [18]. CAS activity was detected for each sample at $420 \mathrm{~nm}$, and relative CAS activity was calculated by subtracting the background from the non-inoculated control and normalising to growth rate. Data are presented as a ratio of the relative CAS activity of the treatment to the control and are representative of biological replicates. Because of the low sensitivity of the CAS assay, batch culture systems were used in order to acquire enough supernatant for the assay.

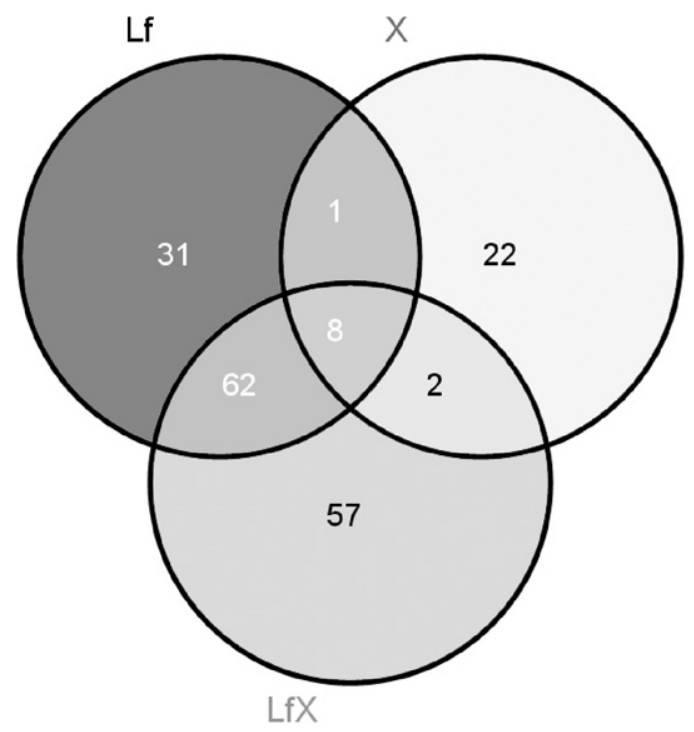

Fig. 2. Venn diagram of differentially expressed genes across the three treatments with either lactoferrin alone (Lf), xylitol alone (X), or lactoferrin and xylitol in combination (LfX). Transcript detection was selected for differential expression of $\geq 1.5$-fold relative to the control sample. 

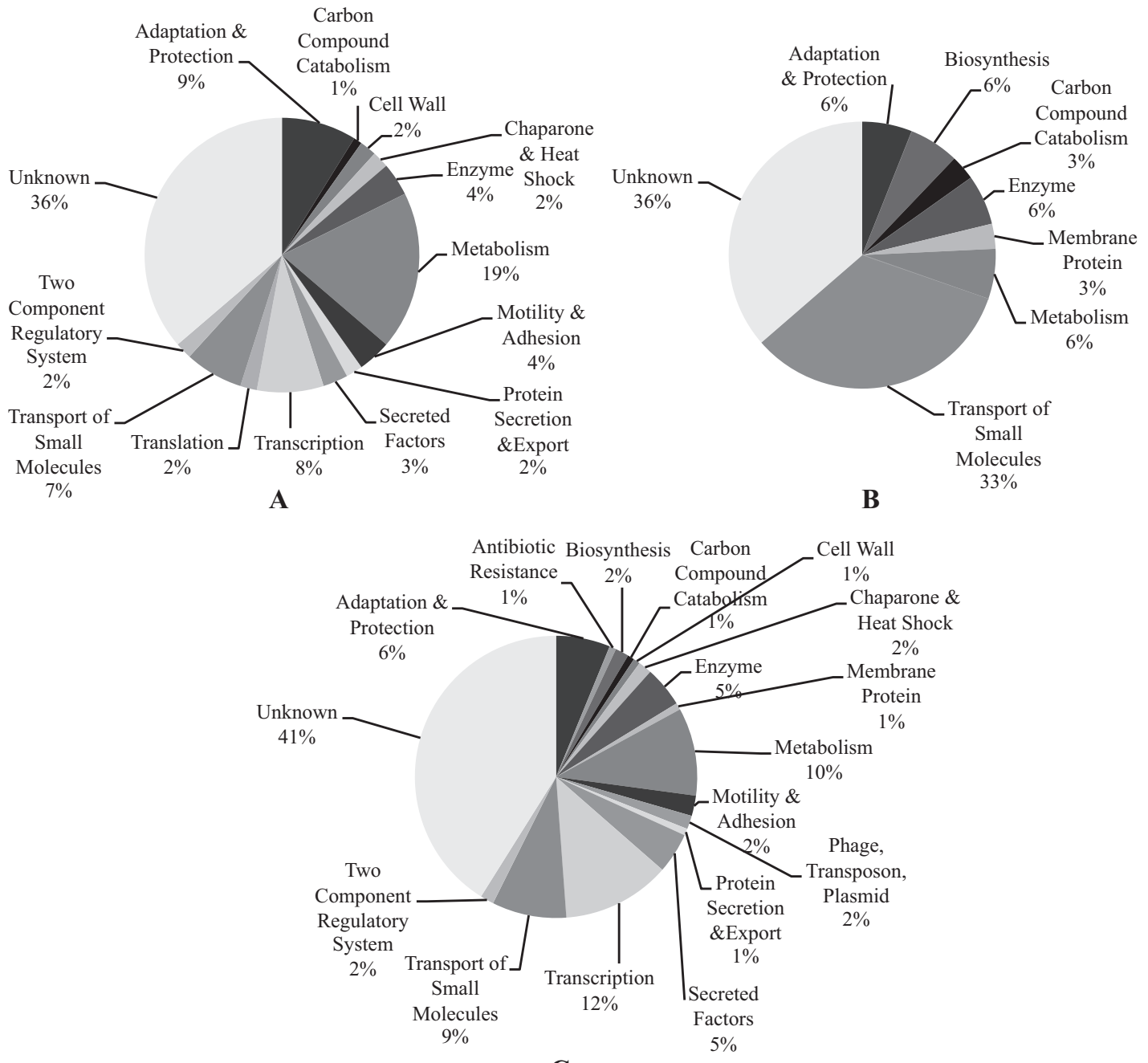

C

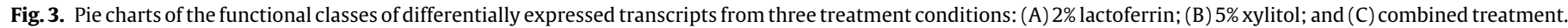

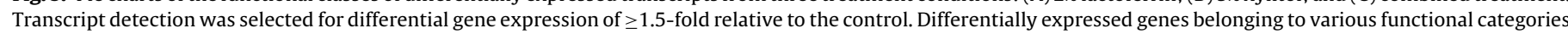
were annotated from the Pseudomonas Genome Database V2 (http://www.pseudomonas.com/).

\subsection{Statistical analysis}

For viability, $\log$ density was $\log _{10}$-transformed and converted to $\log$ reduction relative to the control. Log reduction is the mean $\log$ density of the control minus the mean log density of the treated sample. Relative permeabilised cell number was calculated by the percent area threshold of the treated samples relative to the percent area threshold of the control samples. Statistical analysis was performed using two-tailed unpaired $t$-tests as indicated using GraphPad Prism software (GraphPad Software Inc., San Diego, CA). Data set comparisons with differences at $P<0.05$ were considered statistically significant.

\section{Results}

\subsection{Lactoferrin antimicrobial activity against Pseudomonas} aeruginosa is primarily mediated through iron chelation

In previous work [15], dual treatment of $P$. aeruginosa biofilms with lactoferrin and xylitol resulted in an increase in bacterial cell wall permeabilisation. To demonstrate the antimicrobial component of the combination responsible for this observed membrane destabilisation, further experiments were conducted to detect whether lactoferrin or xylitol destabilised the bacterial membrane.
Using the CDC reactor system, $P$. aeruginosa biofilms were allowed to establish prior to treatment with lactoferrin and/or xylitol. By quantifying the number of bacterial cells staining with a dye that does not cross intact membranes and normalising this number to controls, lactoferrin was determined to be primarily responsible for the membrane permeabilisation observed in the combined treatment, since there was no statistical difference between the lactoferrin-only treatment and the combined treatment (Fig. 1A).

Lactoferrin can both bind endotoxin and chelate iron; however, it remained to be determined which of these binding mechanisms accounts for the ability of lactoferrin to destabilise the membrane of $P$. aeruginosa. To assay for endotoxin-binding ability, lactoferrin containing different levels of endotoxin was assayed for antimicrobial efficacy. When a $\mathrm{CDC}$ reactor was treated with lactoferrin containing low levels of endotoxin, there was a slight difference in the biofilm viability in comparison with biofilms treated with lactoferrin containing high levels of endotoxin (data not shown).

To assay the effect of iron chelation on the antimicrobial efficacy of lactoferrin, $P$. aeruginosa grown in the CDC reactor model was treated with iron-limited lactoferrin (apo-lactoferrin) or with ironsaturated lactoferrin (halo-lactoferrin). Both in the biofilm mode of growth and the planktonic mode of growth there were statistically significant differences in bacterial viability between the apo-lactoferrin-treated samples and the halo-lactoferrin-treated 
samples. Indeed, the $P$. aeruginosa biofilm showed enhanced growth in the presence of halo-lactoferrin (Fig. 1B), suggesting that with iron saturation the bacteria were able to strip some iron from the lactoferrin, thus enhancing bacterial growth. To determine whether the iron chelation of lactoferrin not only contributes to reduced viability but also contributes to membrane permeabilisation, biofilms were harvested and stained for membrane integrity for in situ image analysis. Treatment with apo-lactoferrin results in large numbers of bacterial cells with destabilised membranes compared with treatment with halo-lactoferrin or the untreated control (data not shown).

\subsection{Comparison of differential gene expression between biofilms treated with lactoferrin, xylitol, or lactoferrin and xylitol in combination}

To identify relevant gene expression changes in the microarray analysis, dye swap experiments were performed and differentially expressed genes found in both channels were identified. Only differential gene expression $\geq 1.5 \log$ ratio was selected for further analysis. Of 310 differentially expressed genes, 183 genes were differentially expressed at $\geq 1.5 \mathrm{log}$ ratio in either of the samples. Venn diagram analysis identified eight genes differentially expressed in all three treatments. Interestingly, whilst the lactoferrin-only and xylitol-only samples had closer numbers of unique, differentially expressed genes ( 31 and 22, respectively), the combined treatment had 57 unique, differentially expressed genes. Finally, Venn diagram analysis indicated that the combined treatment resulted in a differential gene expression pattern more similar to the lactoferrinonly treated samples than to the xylitol-only treated samples (70 genes vs. 10 genes, respectively, common to the data sets) (Fig. 2).

Of the 183 differentially expressed genes with $\geq 1.5 \log$ ratio, 74 were identified as hypothetical and therefore had unknown functions. The remaining 109 genes of known function were sorted according to functional class (Fig. 3). In all data sets, a significant number of differentially expressed genes were of unknown function. Of the functionally described genes, the most dynamic functional category for each treatment differed. For the lactoferrinonly treatment the most genetically dynamic functional class was that containing genes associated with metabolism (19\%), and for the xylitol-only treatment the most genetically dynamic functional class was that containing genes associated with the transport of small molecules (33\%). For the combined treatment the most genetically dynamic functional class was that containing genes associated with transcription (12\%).

Differentially regulated transcripts in the combined treatment were sorted according to comparison with microarray analysis of $P$. aeruginosa as deposited in the NCBI GEO. Whilst only a single transcript (napD) associated with biofilm quorum sensing, multiple transcripts associated with environmental iron restriction were differentially regulated. Unexpectedly, these transcripts were downregulated in the combined treatment microarray analysis relative to the control (Table 2).

\subsection{Combined treatment with lactoferrin and xylitol inhibits Pseudomonas aeruginosa expression of the pyochelin biosynthesis pathway}

The surprising findings that transcripts associated with iron restriction were downregulated in the combined treatment microarray led to a closer examination of operons associated with iron restriction. Comparing Table 2 with patterns of expression across operons, the pyochelin biosynthesis operon was found to be differentially expressed. Genes of the pyochelin pathway are responsible for biosynthesis of the siderophore pyochelin in



Fig. 4. Combined treatment of Pseudomonas aeruginosa biofilms with lactoferrin and xylitol inhibits the ability of bacteria to respond to iron stress from lactoferrin through transcriptional regulation of the pyochelin biosynthesis pathway. Quantitative real-time polymerase chain reaction (qPCR) analysis of the genes for the pyochelin biosynthesis operon transcriptional regulator ( $p c h R)$, the salicylate biosynthesis isochorismate synthase enzyme ( $p c h A)$, the pyochelin synthetase enzyme ( $p c h F)$, the Fe(III)-pyochelin outer membrane receptor $(f p t A)$, the biofilmassociated periplasmic nitrate reductase chaperone (napD) and the cytochrome o ubiquinol oxidase $(c y o C)$ is presented. Data are presented as fold change in the transcript relative to the untreated control. Differential transcript expression was normalised to the endogenous $16 S$ rRNA cDNA. Data are representative of repeat experiments.

response to environmental iron restriction. Given the observation that lactoferrin iron chelation is a major antimicrobial mechanism mediated against $P$. aeruginosa biofilms (Fig. 1), the differential gene expression of this operon was of significant interest. Therefore, differential transcript expression of key components of this pathway was validated by real-time qPCR. For comparison with the microarray analysis, the quorum-sensing-associated napD gene and the metabolism-associated $c y o C$ gene were also validated by real-time qPCR (Fig. 4). Whilst the transcriptional regulator of the operon $(p c h R)$ was slightly upregulated, the key first enzyme ( $p c h A)$ and a component of the key final enzyme $(p c h F)$ in the pyochelin biosynthesis pathway were downregulated. Finally, the gene for the pyochelin receptor ( fptA) was minimally repressed compared with the control samples (Fig. 4). As validation of the comparison between the microarray data and the real-time qPCR, the napD and $c y o C$ genes exhibited similar patterns of expression with both methods of analysis (Fig. 4).

\subsection{Xylitol inhibits Pseudomonas aeruginosa response to iron restriction}

To determine whether addition of xylitol to the lactoferrin treatment accounted for the inhibition of siderophore protein production and secretion, $P$. aeruginosa was grown in minimal $\mathrm{KB}$ medium supplemented with either lactoferrin or xylitol. Controls were grown without media supplementation. Siderophore presence in the medium was detected using the CAS activity assay [25]. Over a 48-h course, the growth rate and relative CAS activity were assayed. Whilst $P$. aeruginosa grew more slowly in the presence of lactoferrin, by $48 \mathrm{~h}$ all the cultures had reached similar densities (Fig. 5A). Although little CAS activity was detected at $24 \mathrm{~h}$, by $48 \mathrm{~h}$ a notable difference in CAS activity was observed between the lactoferrin- and xylitol-treated cultures. With lactoferrin treatment, $P$. aeruginosa had nearly 10 -fold greater CAS activity relative to untreated controls by $48 \mathrm{~h}$ post inoculation (Fig. 5B). On the other hand, by $48 \mathrm{~h}$ xylitol treatment had inhibited CAS activity by ca. 3 -fold relative to the untreated controls (Fig. 5B). 
Table 2

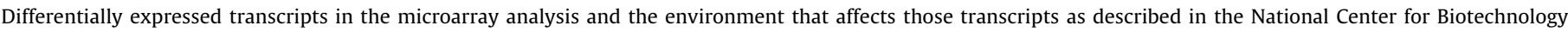
Information (NCBI) Gene Expression Omnibus (GEO).

\begin{tabular}{|c|c|c|c|c|}
\hline Gene & Function & Environment & Fold change & Reference \\
\hline napD & & Quorum induced & 2.043 & Schuster et al. [20] \\
\hline cyoC & Metabolism & $\mathrm{H}_{2} \mathrm{O}_{2}$ repressed & -1.468 & Chang et al. [21] \\
\hline pchF & Iron regulated & $\mathrm{H}_{2} \mathrm{O}_{2}$ repressed & -1.873 & Chang et al. [21] \\
\hline pchA & Iron regulated & $\mathrm{H}_{2} \mathrm{O}_{2}$ repressed & -1.834 & Chang et al. [21] \\
\hline PA0617 & Pyocin & $\mathrm{H}_{2} \mathrm{O}_{2}$ induced & 2.483 & Chang et al. [21] \\
\hline PA4220 & Iron regulated & PQS induced & -1.472 & Bredenbruch et al. [22] \\
\hline pchF & Iron regulated & PQS induced & -1.873 & Bredenbruch et al. [22] \\
\hline pchA & Iron regulated & PQS induced & -1.834 & Bredenbruch et al. [22] \\
\hline PA4220 & Oxidative stress & PQS induced & -1.472 & Bredenbruch et al. [22] \\
\hline pchF & Oxidative stress & PQS induced & -1.873 & Bredenbruch et al. [22] \\
\hline pchA & Oxidative stress & PQS induced & -1.834 & Bredenbruch et al. [22] \\
\hline cyoC & Oxidative stress & Low oxygen/nitrogen repressed & -1.468 & Alvarez-Ortega and Harwood [23] \\
\hline napD & Oxidative stress & Low oxygen induced & 2.043 & Alvarez-Ortega and Harwood [23] \\
\hline PA0617 & Oxidative stress & Nitrogen induced & 2.483 & Alvarez-Ortega and Harwood [23] \\
\hline PA4738 & Oxidative stress & Low oxygen induced & -1.621 & Alvarez-Ortega and Harwood [23] \\
\hline PA2034 & & Iron restriction induced & -1.691 & Ochsner et al. [24] \\
\hline$p c h F$ & & Iron restriction induced & -1.873 & Ochsner et al. [24] \\
\hline PA2451 & & Iron restriction induced & -1.599 & Ochsner et al. [24] \\
\hline PA2452 & & Iron restriction induced & -1.655 & Ochsner et al. [24] \\
\hline PA2468 & & Iron restriction induced & -1.82 & Ochsner et al. [24] \\
\hline PA4895 & & Iron restriction induced & -2.314 & Ochsner et al. [24] \\
\hline PA3900 & & Iron restriction induced & -1.589 & Ochsner et al. [24] \\
\hline PA4708 & & Iron restriction induced & -1.531 & Ochsner et al. [24] \\
\hline PA5150 & & Iron restriction induced & -1.86 & Ochsner et al. [24] \\
\hline PA1134 & & Iron restriction induced & -1.666 & Ochsner et al. [24] \\
\hline cyoC & & Iron restriction induced & -1.468 & Ochsner et al. [24] \\
\hline
\end{tabular}

PQS, Pseudomonas quinolone signal.

\section{Discussion}

Although chronic wounds typically only affect people over the age of 60 years [26], this sector of the population is growing. Whilst the incidence of chronic wounds is ca. 2\% [27], this number has more than doubled since 2004 [28] and is expected to increase with the ageing population. Contaminating bacterial biofilm appears to be a primary factor in non-healing wounds, which confirms the need for biofilm-targeted strategies to manage chronic wounds [5]. In a recent study, use of the innate immune protein lactoferrin in combination with the rare sugar alcohol xylitol to treat $P$. aeruginosa biofilms in vitro was demonstrated [15]. Whilst combined treatment proved more effective against $P$. aeruginosa biofilms compared with either treatment alone, the mechanism of co-operative interaction between these two antimicrobials remained elusive. Data presented in this study demonstrate a potential model for the molecular mechanism of this co-operation.

Lactoferrin is produced by cells of the innate immune system and has recently been demonstrated to be efficacious in inhibiting bacterial biofilm formation of periodontal pathogens [29], cystic fibrosis pathogens $[15,30]$ and biofilm-associated chronic rhinosinusitis [31]. Lactoferrin is well known to damage the outer membrane of Gram-negative bacteria [32] and both to chelate iron [33] and to bind endotoxin [34]. This study demonstrates that lactoferrin exerts antimicrobial activity against $P$. aeruginosa biofilms primarily through iron chelation (Fig. 1). This iron-binding capacity results in destabilisation of the bacterial membrane as demonstrated by membrane permeabilisation (data not shown). Whilst the mechanism by which iron chelation destabilises the bacterial membrane remains to be directly determined, correlative analysis indicated that lactoferrin mediates osmotic damage to the bacterial membrane and that this mechanism is inhibited by iron saturation [35]. Based on the findings demonstrated here, it is probable that lactoferrin and other antimicrobials may interact in a beneficial manner when used in combination. One major obstacle in antimicrobial treatment is penetration of the antimicrobial into the bacterial cell. This a particular problem with Gram-negative bacteria such as $P$. aeruginosa [36]. Destabilisation of the bacterial membrane by lactoferrin demonstrated here might enhance the efficacy of other
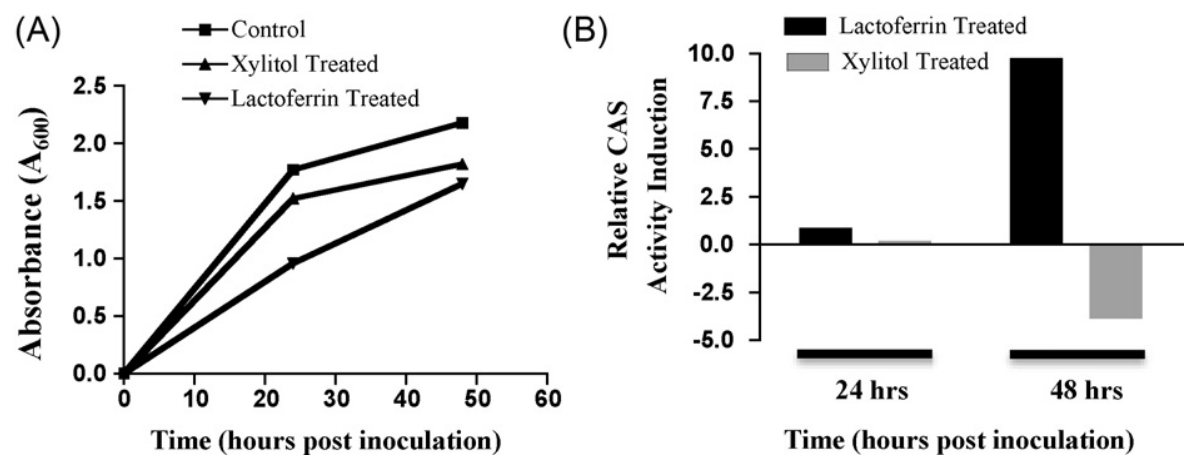

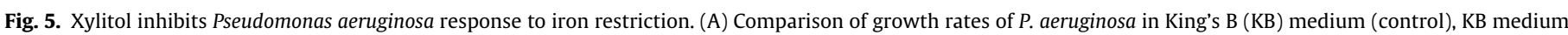

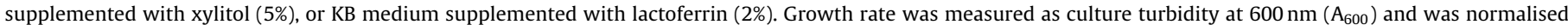

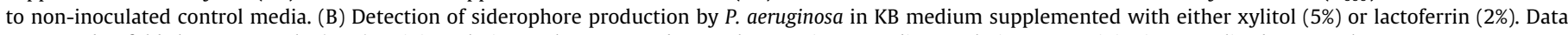
presented as fold chrome azurol S (CAS) activity relative to the untreated control grown in KB medium. Relative CAS activity is normalised to growth rate. 


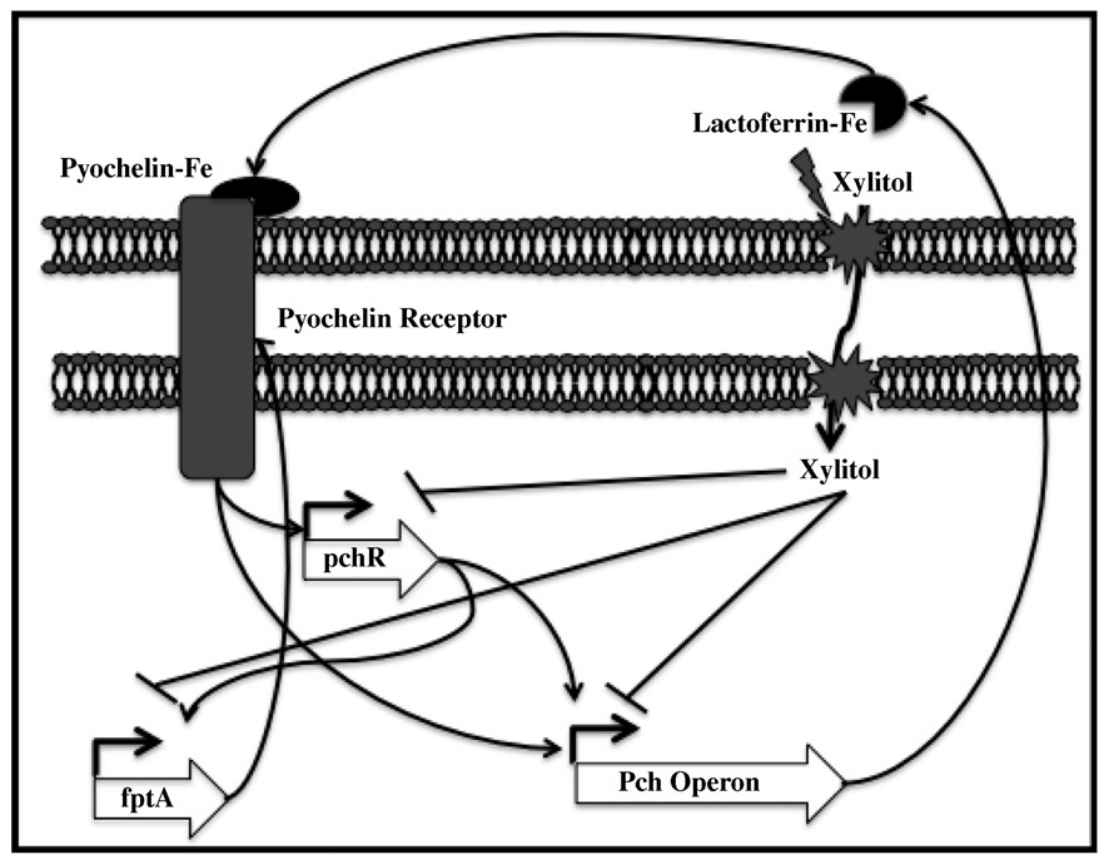

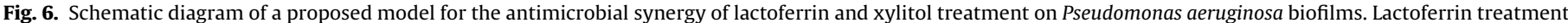

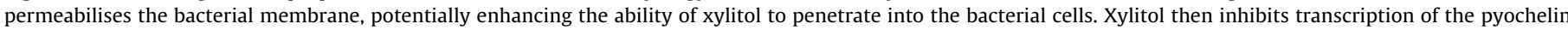
biosynthesis pathway and the bacteria are thus unable to respond to lactoferrin iron chelation, enhancing the antimicrobial activity of lactoferrin.

antimicrobials by improving penetration into the bacterial cell. Exploration of this potential for interaction between lactoferrin and other antimicrobials remains an interesting area for further pursuit.

Xylitol has also previously been demonstrated to inhibit bacterial biofilms. The antibiofilm properties of xylitol have primarily been demonstrated with dental-associated pathogens and have been shown to be effective at inhibiting bacterial adhesion and growth [37] as well as the expression of metabolising enzymes required for biofilm formation of cariogenic streptococci [38]. Biofilm growth inhibition by xylitol has also been demonstrated in an in vitro six-species oral biofilm model [39] and to be effective against colonisation by Staphylococcus aureus of atopic dry skin [40]. Finally, previous studies have demonstrated that xylitol is moderately effective against $P$. aeruginosa biofilms grown in vitro [15].

Synergistic interactions have previously been demonstrated for both lactoferrin and xylitol. For example, synergistic interactions have been demonstrated for xylitol used in combination with benzethonium chloride [41], chlorhexidine [37] and farnesol [40]. For lactoferrin, synergism has been demonstrated when combined with secretory immunoglobulin A $[42,43]$ as well as lysozyme and vancomycin [44]. Finally, co-operative inhibition of $P$. aeruginosa biofilms by lactoferrin and xylitol has been demonstrated [15]. Despite these intriguing interactions, little work has been done to characterise this co-operative effect on a molecular and cellular level.

Utilising transcriptomic analysis, this study demonstrated that combined lactoferrin and xylitol treatment of $P$. aeruginosa biofilms resulted in numerous biological effects at the molecular level. Although the transcriptomic analysis provides many interesting pathways for further investigation, a complete pursuit of each component of interest is beyond the scope of the current study; therefore, this study focused on a single operon of particular interest for which the transcriptomic analysis identified differential gene expression. From the 183 differentially expressed transcripts, the operon encoding the components of pyochelin biosynthesis was chosen for further evaluation because of the relationship between lactoferrin and pyochelin reported in the literature [45-47]. Although little work has been done demon- strating the role of pyochelin in P. aeruginosa biofilms [48], this siderophore clearly plays an important role in $P$. aeruginosa iron acquisition during environmental conditions of iron restriction (reviewed in [49]). When iron is restricted in the environment, the iron-responsive transcription factor PchR activates expression of the components of pyochelin biosynthesis. Regulation of pyochelin synthesis is on a positive feedback loop as iron-bound pyochelin activates the pyochelin receptor, which then further stimulates expression of the pch operon [50].

To examine this adaptive pathway in response to treatment, real-time qPCR was performed on the pathway regulator, key biosynthesis enzymes and the pathway receptor. Surprisingly, treatment with lactoferrin and xylitol in combination resulted in mild upregulation of the transcription regulator $p c h R$ but significant downregulation of two major enzymes of the pyochelin biosynthesis pathway ( $p c h A$ and $p c h F$ ). Finally, expression of the pyochelin receptor was negligible compared with the untreated controls (Fig. 4).

To verify further that xylitol was the component of the combined treatment that interferes with the ability of $P$. aeruginosa to respond to lactoferrin iron chelation by producing siderophores, lactoferrin and xylitol treatment were assayed independently for the production of siderophores in culture using the CAS activity assay [25]. When normalised to growth rates and control media, siderophore production in the lactoferrin-treated samples increased relative to the untreated samples, whilst siderophore production in the xylitol-treated samples decreased relative to the untreated samples (Fig. 5B). These data demonstrate that whilst treatment with lactoferrin enhances siderophore production (as would be expected since lactoferrin is an iron chelator), treatment with xylitol inhibits siderophore production relative to untreated $P$. aeruginosa cultures.

Whilst iron chelation was demonstrated to be of key importance to lactoferrin permeabilisation of the bacterial membrane, microarray analysis and real-time qPCR identified regulation of the pyochelin biosynthesis pathway by xylitol to be of key importance. With xylitol inhibiting a crucial element of $P$. aeruginosa adaptation to iron restriction, the bacteria may become more sensitive to membrane instability mediated by lactoferrin. Although adaptation 
to xylitol has been demonstrated to include lack of retention of xylitol by treated bacteria, lactoferrin destabilisation of the membrane may enhance the ability of xylitol to penetrate into the bacterial cell (Fig. 6). Although the transcriptomic analysis warrants further investigation, this study demonstrates support for a model for a molecular mechanism that contributes to the co-operative antimicrobial effect of lactoferrin and xylitol on $P$. aeruginosa biofilms.

\section{Acknowledgment}

Dr Randy Wolcott (Southwest Regional Wound Care Center, Lubbock, TX) kindly supplied the clinical wound isolates.

Funding: This work was supported by Glanbia Nutritionals, Inc. (Monroe, WI).

Competing interests: LSW is employed by Glanbia Nutritionals Inc. and provided the Bioferrin ${ }^{\circledR}$ used in these experiments.

Ethical approval: Not required.

\section{References}

[1] James GA, Swogger E, Wolcott R, Pulcini E, Secor P, Sestrich J, et al. Biofilms in chronic wounds. Wound Repair Regen 2008;16:37-44.

[2] Konturek PC, Brzozowski T, Konturek SJ, Kwiecien S, Dembinski A, Hahn EG. Influence of bacterial lipopolysaccharide on healing of chronic experimental ulcer in rat. Scand J Gastroenterol 2001;36:1239-47.

[3] Power C, Wang JH, Sookhai S, Street JT, Redmond HP. Bacterial wall products induce downregulation of vascular endothelial growth factor receptors on endothelial cells via a CD14-dependent mechanism: implications for surgical wound healing. J Surg Res 2001;101:138-45.

[4] Costerton JW, Stewart PS, Greenberg EP. Bacterial biofilms: a common cause of persistent infections. Science 1999;284:1318-22.

[5] Rhoads DD, Wolcott RD, Percival SL. Biofilms in wounds: management strategies. J Wound Care 2008; 17:502-8.

[6] Donlan RM, Costerton JW. Biofilms: survival mechanisms of clinically relevant microorganisms. Clin Microbiol Rev 2002;15:167-93.

[7] Domingue Sr GJ, Hellstrom WJ. Prostatitis. Clin Microbiol Rev 1998;11:604-13.

[8] Nickel JC, Costerton JW. Coagulase-negative staphylococcus in chronic prostatitis. J Urol 1992;147:398-400 [discussion 400-1].

[9] Govan JR, Deretic V. Microbial pathogenesis in cystic fibrosis: mucoid Pseu domonas aeruginosa and Burkholderia cepacia. Microbiol Rev 1996;60:539-74.

[10] Gjodsbol K, Christensen JJ, Karlsmark T, Jorgensen B, Klein BM, Krogfelt KA Multiple bacterial species reside in chronic wounds: a longitudinal study. Int Wound J 2006;3:225-31.

[11] Dowd SE, Sun Y, Secor PR, Rhoads DD, Wolcott BM, James GA, et al. Survey of bacterial diversity in chronic wounds using pyrosequencing, DGGE, and full ribosome shotgun sequencing. BMC Microbiol 2008;8:43.

[12] Valenti P, Berlutti F, Conte MP, Longhi C, Seganti L. Lactoferrin functions: current status and perspectives. J Clin Gastroenterol 2004;38(6 Suppl.):S127-9.

[13] Singh PK, Parsek MR, Greenberg EP, Welsh MJ. A component of innate immunity prevents bacterial biofilm development. Nature 2002;417:552-5.

[14] Granström TB, Izumori K, Leisola M. A rare sugar xylitol. Part II: biotechnolog ical production and future applications of xylitol. Appl Microbiol Biotechnol 2007;74:273-6.

[15] Ammons MC, Ward LS, Fisher ST, Wolcott RD, James GA. In vitro susceptibility of established biofilms composed of a clinical wound isolate of Pseudomonas aeruginosa treated with lactoferrin and xylitol. Int J Antimicrob Agents 2009;33:230-6.

[16] Kim J, Pitts B, Stewart PS, Camper A, Yoon J. Comparison of the antimicrobia effects of chlorine, silver ion, and tobramycin on biofilm. Antimicrobial Agents Chemother 2008;52:1446-53.

[17] Puckette MC, Tang Y, Mahalingam R. Transcriptomic changes induced by acute ozone in resistant and sensitive Medicago truncatula accessions. BMC Plant Biol 2008;8:46.

[18] Trivedi P, Pandey A, Palni LM. In vitro evaluation of antagonistic properties of Pseudomonas corrugata. Microbiol Res 2008;163:329-36.

[19] Raaska L, Sillanpaa J, Sjoberg AM, Suihko ML. Potential microbiological hazards in the production of refined paper products for food applications. J Ind Microbiol Biotechnol 2002;28:225-31.

[20] Schuster M, Hawkins AC, Harwood CS, Greenberg EP. The Pseudomonas aeruginosa RpoS regulon and its relationship to quorum sensing. Mol Microbiol 2004;51:973-85.

[21] Chang W, Small DA, Toghrol F, Bentley WE. Microarray analysis of Pseudomonas aeruginosa reveals induction of pyocin genes in response to hydrogen peroxide. BMC Genomics 2005;6:115.
[22] Bredenbruch F, Geffers R, Nimtz M, Buer J, Haussler S. The Pseudomonas aeruginosa quinolone signal (PQS) has an iron-chelating activity. Environ Microbiol 2006;8:1318-29.

[23] Alvarez-Ortega C, Harwood CS. Responses of Pseudomonas aeruginosa to low oxygen indicate that growth in the cystic fibrosis lung is by aerobic respiration. Mol Microbiol 2007;65:153-65.

[24] Ochsner UA, Wilderman PJ, Vasil AI, Vasil ML. GeneChip expression analysis of the iron starvation response in Pseudomonas aeruginosa: identification of novel pyoverdine biosynthesis genes. Mol Microbiol 2002;45:1277-87.

[25] Schwyn B, Neilands JB. Universal chemical assay for the detection and determination of siderophores. Anal Biochem 1987:160:47-56.

[26] Mustoe T. Understanding chronic wounds: a unifying hypothesis on their pathogenesis and implications for therapy. Am J Surg 2004;187: 65S-70S.

[27] Bjarnsholt T, Kirketerp-Møller K, Jensen PØ, Madsen KG, Phipps R, Krogfelt K, et al. Why chronic wounds will not heal: a novel hypothesis. Wound Repair Regen 2008;16:2-10.

[28] Crovetti G, Martinelli G, Issi M, Barone M, Guizzardi M, Campanati B, et al. Platelet gel for healing cutaneous chronic wounds. Transfus Apher Sci 2004;30:145-51.

[29] Wakabayashi H, Yamauchi K, Kobayashi T, Yaeshima T, Iwatsuki K, Yoshie H. Inhibitory effects of lactoferrin on growth and biofilm formation of Porphyromonas gingivalis and Prevotella intermedia. Antimicrob Agents Chemother 2009;53:3308-16.

[30] O'May CY, Sanderson K, Roddam LF, Kirov SM, Reid DW. Iron-binding compounds impair Pseudomonas aeruginosa biofilm formation, especially under anaerobic conditions. J Med Microbiol 2009;58:765-73.

[31] Psaltis AJ, Wormald PJ, Ha KR, Tan LW. Reduced levels of lactoferrin in biofilmassociated chronic rhinosinusitis. Laryngoscope 2008;118:895-901.

[32] Ellison 3rd RT, Giehl TJ, LaForce FM. Damage of the outer membrane of enteric Gram-negative bacteria by lactoferrin and transferrin. Infect Immun 1988:56:2774-81.

[33] Singh PK. Iron sequestration by human lactoferrin stimulates $P$. aeruginosa surface motility and blocks biofilm formation. Biometals 2004;17: 267-70.

[34] Appelmelk BJ, An YQ, Geerts M, Thijs BG, de Boer HA, MacLaren DM, et al. Lactoferrin is a lipid A-binding protein. Infect Immun 1994;62:2628-32.

[35] Ellison 3rd RT, Giehl TJ. Killing of Gram-negative bacteria by lactoferrin and lysozyme. J Clin Invest 1991;88:1080-91.

[36] Savage PB. Multidrug-resistant bacteria: overcoming antibiotic permeability barriers of Gram-negative bacteria. Ann Med 2001;33:167-71.

[37] Decker EM, Maier G, Axmann D, Brecx M, von Ohle C. Effect of xylitol/chlorhexidine versus xylitol or chlorhexidine as single rinses on initial biofilm formation of cariogenic streptococci. Quintessence Int 2008;39: $17-22$.

[38] Shemesh M, Tam A, Feldman M, Steinberg D. Differential expression profiles of Streptococcus mutans $\mathrm{ftf}$, gtf and vicR genes in the presence of dietary carbohydrates at early and late exponential growth phases. Carbohydr Res 2006;341:2090-7.

[39] Badet C, Furiga A, Thebaud N. Effect of xylitol on an in vitro model of oral biofilm. Oral Health Prev Dent 2008;6:337-41.

[40] Katsuyama M, Ichikawa H, Ogawa S, Ikezawa Z. A novel method to control the balance of skin microflora. Part 1. Attack on biofilm of Staphylococcus aureus without antibiotics. J Dermatol Sci 2005;38:197-205.

[41] Ichikawa T, Yano Y, Fujita Y, Kashiwabara T, Nagao K. The enhancement effect of three sugar alcohols on the fungicidal effect of benzethonium chloride toward Candida albicans. J Dent 2008;36:965-8.

[42] Stephens S, Dolby JM, Montreuil J, Spik G. Differences in inhibition of the growth of commensal and enteropathogenic strains of Escherichia coli by lactotransferrin and secretory immunoglobulin A isolated from human milk. Immunology 1980;41:597-603.

[43] Dolby JM, Stephens S. Antibodies to Escherichia coli O antigens and the invitro bacteriostatic properties of human milk and its IgA. Acta Paediatr Scand 1983;72:577-82.

[44] Leitch EC, Willcox MD. Lactoferrin increases the susceptibility of S. epidermidis biofilms to lysozyme and vancomycin. Curr Eye Res 1999;19:12-19.

[45] Husson MO, Legrand D, Spik G, Leclerc H. Iron acquisition by Helicobacter pylori: importance of human lactoferrin. Infect Immun 1993;61:2694-7.

[46] Yang H, Kooi CD, Sokol PA. Ability of Pseudomonas pseudomallei malleobactin to acquire transferrin-bound, lactoferrin-bound, and cell-derived iron. Infect Immun 1993:61:656-62.

[47] Sriyosachati S, Cox CD. Siderophore-mediated iron acquisition from transferrin by Pseudomonas aeruginosa. Infect Immun 1986;52:885-91.

[48] Harjai K, Khandwahaa RK, Mittal R, Yadav V, Gupta V, Sharma S. Effect of pH on production of virulence factors by biofilm cells of Pseudomonas aeruginosa. Folia Microbiol (Praha) 2005;50:99-102.

[49] Poole K, McKay GA. Iron acquisition and its control in Pseudomonas aeruginosa: many roads lead to Rome. Front Biosci 2003;8:d661-86.

[50] Gaille C, Reimmann C, Haas D. Isochorismate synthase (PchA), the first and rate-limiting enzyme in salicylate biosynthesis of Pseudomonas aeruginosa. J Biol Chem 2003;278:16893-8. 